ISSN 1997-342X (Online), ISSN 1991-8631 (Print)

Original Paper http://ajol.info/index.php/ijbcs http://indexmedicus.afro.who.int

\title{
Comment les aires protégées structurent les écosystèmes des périphéries
}

\author{
Ngaryam BENOUDJITA* et Alain DJINET IGNASSOU \\ Département des Sciences de la Vie et de la Terre, Ecole Normale Supérieure de Bongor, Tchad. \\ ${ }^{*}$ Corresponding auteur; E-mail: ben_nga@yahoo.fr, BP: 15 Bongor, Tchad.
}

\section{RESUME}

Cette étude menée a pour but de montrer l'influence des aires protégées sur la structuration des écosystèmes périphériques. Pour cela, une étude diachronique par images satellitaires Landsat de 1985 à 2013 a été faite et une collecte de données de terrain a été réalisée dans les villages autour du Parc National de Manda dans le méridien tchadien en vue d'apprécier la gestion de la biodiversité de leur entourage. Des résultats, il ressort que la création du parc a favorisé la délocalisation de certains villages provoquant une concentration démographique ayant pour corollaire une dégradation accélérée des ressources accessibles due aux effets de reports. Cette dégradation est ici mesurée par la modification de la structure des écosystèmes adjacents et du mode d'accès habituel aux ressources naturelles des communautés locales. L'analyse de la structure verticale des écosystèmes montre que la surexploitation affecte plus la strate arborée réduisant ainsi les écosystèmes à 3 ou 2 strates selon les endroits (la strate arborée est passée de 19,53\% en 1985 à 7,58\% en 2013, la strate arbustive a suivi aussi la même courbe descendante passant de $20,72 \%$ à $7,14 \%$ ). A contrario, les mosaïques de champs et jachères ont connu une augmentation de leur superficie (de 17,24\% à 54,42\%). La modification de la structure horizontale est marquée par l'hétérogénéité spatiale de la biocénose. Les résultats obtenus constituent une interpellation des gestionnaires de l'environnement que l'avenir de la conservation des ressources biologiques ne se joue pas seulement dans les aires protégées. L'intégration des zones non protégées dans un plan de gestion rationnelle du territoire pourrait être un gage de conservation à long terme.

(C) 2017 International Formulae Group. All rights reserved.

Mots clés : Parc, biodiversité, Manda, structure d'écosystème.

\section{How protected areas structure peripheral ecosystems}

\begin{abstract}
The aim of this study is to show the influence of protected areas on the structuring of peripheral ecosystems. For this purpose, a diachronic study using Landsat satellite imagery from 1985 to 2013 was carried out and a field data collection was carried out in the villages around the Manda National Park in the Chadian meridian in order to assess the management of biodiversity of their entourage. The results show that the creation of the park has favored the delocalisation of villages causing a demographic concentration resulting in an accelerated degradation of available resources due to the effects of carryovers. This degradation is measured by changes in the structure of adjacent ecosystems and the usual access to the natural resources of local communities. Analysis of the vertical structure of ecosystems shows that overexploitation affects the tree stratum, reducing ecosystems to 3 or 2 strata depending on the location (the tree stratum decreased from $19.53 \%$ in 1985 to $7.58 \%$ in 2013 , the shrub stratum also followed the same downward curve from $20.72 \%$ to
\end{abstract}


7.14\%). On the contrary, the mosaics of fields and fallows have increased their area (from $17.24 \%$ to $54.42 \%$ ). The modification of the horizontal structure is marked by the spatial heterogeneity of the biocenosis. The results obtained constitute an inquiry by environmental managers that the future of the conservation of biological resources is not only played out in protected areas. The integration of unprotected areas into a rational management plan for the territory could be a guarantee of long-term.

(C) 2017 International Formulae Group. All rights reserved.

Keywords: Park, biodiversity, Manda, ecosystem structure.

\section{INTRODUCTION}

La mise sous cloche des ressources naturelles ou la sanctuarisation de certains espaces ne date pas du Sommet de la Terre tenu à Rio de Janeiro en 1992 qui a vu, à l'issue des assises, la naissance de la convention sur la conservation de la Diversité Biologique (Desponds, 2012). Cette convention non seulement a popularisé le concept de «biodiversité » mais a explosé la création des aires protégées dans le monde (Gunnell, 2009). Les espaces protégés traditionnels datent de l'antiquité et sont l'œuvre des chefs coutumiers et certaines communautés comme des sites sacrés dédiés aux rites culturels ou des réserves des ressources stratégiques (Gauthier-Clerc et al., 2014). La première aire protégée de création formelle de l'époque moderne est le parc de Yellowstone des Etats Unis d'Amérique en 1872 (Faurie et al., 2012). Dans la convention de 1992, la biodiversité est conservée pour une « utilisation durable de ses éléments et le partage juste et équitable des avantages découlant de l'exploitation des ressources génétiques »(Arnould et Simon, 2007). Il apparait clairement que les objectifs de la convention visent la durabilité de l'exploitation des ressources (Ngaryam, 2016). Ce qui augure bien l'intérêt accordé à la satisfaction des besoins de génération à venir. Cette nouvelle vision est un cadre de réflexion qui oblige l'humanité à changer de point de vue relatif au développement (Arne, 2008). Il ne s'agit plus de croire à la disponibilité illimitée des ressources dans le progrès continu et dans la nécessité de la croissance (Barbault et al., 2004).

Au-delà des raisons qui ont présidé leur genèse et les approches de leur gestion, les aires protégées ont des impacts avérés sur leurs périphéries (Ngaryam, 2016). C'est pourquoi la question est de savoir : comment le changement s'opère dans les écosystèmes lorsqu'une aire protégée est créée ?

L'objectif de cette étude est de montrer que l'influence des espaces protégés sur leurs périphéries peut se mesurer à travers la structuration des écosystèmes adjacents (Manceron, 2011; Sandjong Sani et al., 2013). Pour mettre en évidence la structuration de l'espace provoquée par la création des aires protégées, une étude de cas est réalisée autour du Parc National de Manda au Tchad en Afrique Centrale. Un parc de création coloniale en 1951 comme Réserve de Faune Régionale puis Réserve Territoriale en 1953 sous l'autorité coloniale. Elle est érigée en Parc National en 1965 précisément le 19 mars par décret $n^{\circ} 56 /$ EFPC par le nouveau Etat indépendant. La superficie du parc de Manda est de 114000 ha.

L'étude de cette aire protégée est bâtie sur la connaissance des impacts générés par ce parc national ; l'appréhension des populations; l'état des écosystèmes aux alentours des aires protégées par observation de visu et satellitaires; la profondeur de ces impacts et leur ampleur, etc. ont montré comment une aire protégée peut structurer les écosystèmes périphériques (Ngaryam, 2016).

Dans cette optique, cet article, après déclinaisons des concepts convoqués, une 
brève description du parc national de Manda et de la méthodologie de recherche, la présentation des éléments structurants qui au demeurant ont donné une nouvelle structure aux écosystèmes adjacents sera faite.

Une structure est déclinée par le Dictionnaire Larousse illustré (2005) comme « un ensemble de parties concrètes ou abstraites d'un ensemble arrangées de manière cohérente et en est la caractéristique permanente ou relativement stable ». Autrement dit, la structure d'un écosystème est la disposition des systèmes complexes qui relèvent de l'inféodation de biocénose dans le biotope conférant une architecture caractéristique. En effet, les différents organismes végétaux et animaux de dimensions très variables occupent le plus souvent des espaces bien définis (Dufumier, 2006). Ainsi deux types principaux de structures des écosystèmes sont distinguées : structure verticale (stratification de la biocénose) et structure horizontale appelée aussi hétérogénéité spatiale de la biocénose (Dajoz, 2006).

Dans la structure verticale, les différentes strates se différencient comme suit: la strate cryptogamique, la strate herbacée, la strate arbustive et la strate arborescente en ce qui concerne la composante végétale de la biocénose (Faurie et al., 2012). A cette stratification végétale s'ajoute celle des animaux qui renferme des espèces liées au sol et les oiseaux, les insectes et les mammifères occupant les différents niveaux de strates végétales. En outre, dans le sol, l'étagement des racines donne une autre structure souterraine (Barbault, 2004).

Ces structures se mettent en place naturellement en fonction des facteurs écologiques du milieu tels que la nature du sol, les conditions climatiques et biologiques. Avec les activités de développement du monde contemporain, ces structures sont fortement modifiées (Dovonou-Vinagbè et Chouinard, 2009; Niang, 2009 in Aissatou
Thiam Ndong, 2015). Dans la présente étude, ce sont les transformations des structures des écosystèmes des zones périphériques induites par la création du parc qui sont recherchées. Les facteurs générateurs de structures d'écosystèmes agissent en provoquant de micro-successions d'un type de structure par un autre type (Djego et al., 2011).

En tant qu'unité écologique de base, un écosystème désigne l'ensemble constitué par le biotope et la biocénose en constante interaction l'une avec l'autre (Ramade, 2008).

Dans le contexte de cette étude, la réflexion se focalise sur la structure des écosystèmes de la périphérie des aires protégées et notamment celle du parc national de Manda en Afrique centrale précisément au Tchad.

\section{MATERIEL ET METHODES Description du site d'étude}

Le site de l'étude est la périphérie du Parc National de Manda (PNM) situé dans le méridien tchadien dans la région du Moyen Chari à une trentaine de kilomètre de la ville de Sarh. Cet espace protégé couvre une superficie de 114000 ha après élargissement de ses limites en 1967. La Figure 1 le situe dans un bassin hydrographique au Sud du Tchad. Le parc renferme en termes de richesses faunistiques d'éléphants ; de céphalophes à flanc roux, de cobes de roseaux, de phacochères, d'autruches, de buffles, de léopards, de girafes, etc. au début de la création du parc. Une bonne partie de cette faune a disparu de nos jours notamment les éléphants, les buffles, les lions, etc. L'Eland de Derby (Taurotragus derbianus de la famille des Bovidés) qui a suscité la création du parc, « a disparu du PNM à partir de 1980».

La végétation est en majorité des savanes arborées et arbustives qui hébergent les Gardenia erubescens, Detarium microcarpum, Combretum glutinosum, Prosopis africana, Piliostigma reticulatum, 
Daniella oliveri, Butyrospermum parkii, Piliostigma thonningii ; Terminalia glaucescens ; Grewia mollis, Khaya senegalensis, etc (Djirra, 2011). Cette végétation renferme les bosquets et les forêts avec une alternance de forêts claires, de savanes arbustives et savanes arborées selon les endroits.

\section{Echantillonnage et collecte des données}

L'objectif principal de ce travail est d'apprécier la gestion de la biodiversité à travers les effets des aires protégées sur les ressources biologiques et les milieux abiotiques qui les entourent, en prenant le cas du parc national de Manda au Tchad. Ainsi pour atteindre cet objectif, la méthode de travail consiste en une collecte de données bibliographiques, les entrevues auprès des habitants des villages adjacents, les observations de terrain et l'interprétation des images satellitaires.

Entretiens avec les membres des communautés locales

$\mathrm{Au}$ total, 14 villages adjacents situés dans le pourtour du parc sont concernés par l'étude. Ces villages sont: Guéré, Djoli1, Djoli2, Talia, Sanglé, Boundourou, Moro, Koutou, Niellim, Léabla, Kokaga, Maïrom, Manda et Balimba. Ces villages sont localisés sur la Figure 2 à l'exception de quelques-uns.

Lors de ces entretiens qui se sont déroulés entre 2012 et 2014, deux techniques de collectes de données ont été combinées : l'interview semi-directive à l'aide d'un guide d'entretien adressée aux grands acteurs comme les agriculteurs (19), les pécheurs (07), les éleveurs (11) (nomades et sédentaires) et 7 chefs coutumiers ( 1 chef d'initiation, 4 chefs de terres et 2 Mbang) soient au total 43 acteurs issus des communautés locales témoins du changement de structure des écosystèmes et subissant ses effets. Un autre guide d'entretien est administré au personnel administratif des services déconcentrés de l'Etat tchadien ainsi que des coopératives et les équipes des projets de développement intervenant dans la zone du Parc National de Manda. En l'occurrence, 12 personnes sont interrogées. Les observations directes sur l'état des écosystèmes adjacents avec des relevés points géographiques à l'aide d'un GPS (Global Positioning System) de marque Garmin ont permis de faire une comparaison avec les images satellitaires.

\section{Etude diachronique par images satellitaires}

L'approche paysagère par photointerprétation est une démarche comparative spatio-temporelle. Cette comparaison diachronique permet de se rendre compte des mutations qui se sont succédées dans les écosystèmes des périphéries puis de faire une comparaison entre les écosystèmes de l'intérieur du parc avec ceux des zones adjacentes.

Les images satellitaires utilisées sont celles de 1985, 1995, 2005 et 2013; notamment celles du mois d'octobre qui marque la fin de la saison des pluies dans la région d'étude. A cette époque, il y a moins de nuages et les champs ainsi que les autres paysages apparaissent clairement sur les images satellitaires; ce que les autres mois plus pluvieux ne permettent pas d'observer à cause de la densité élevée des nuages. Les images satellitaires utilisées sont de types Landsat UTM Zone 34 N WGS 84 téléchargées sur Google Earth. Ensuite, elles sont traitées avec le logiciel ArcGis avec l'appui technique du Centre National de Recherche pour le Développement (CNRD) afin de mettre en exergue l'évolution des différents paysages au fil du temps sous l'effet des pressions anthropiques et du bouleversement occasionné par la création du parc. 


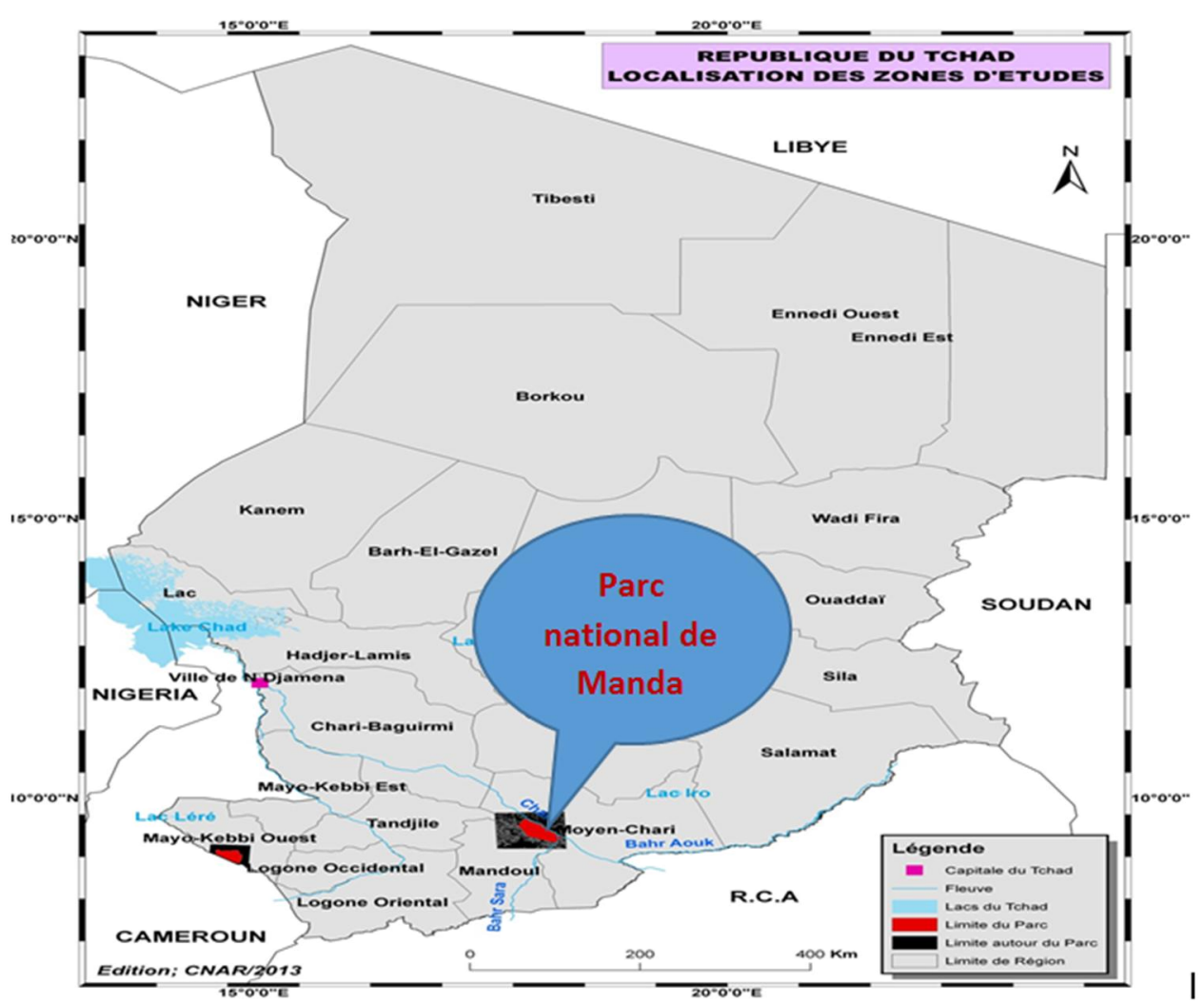

Figure 1 : Carte de localisation du site d'étude.

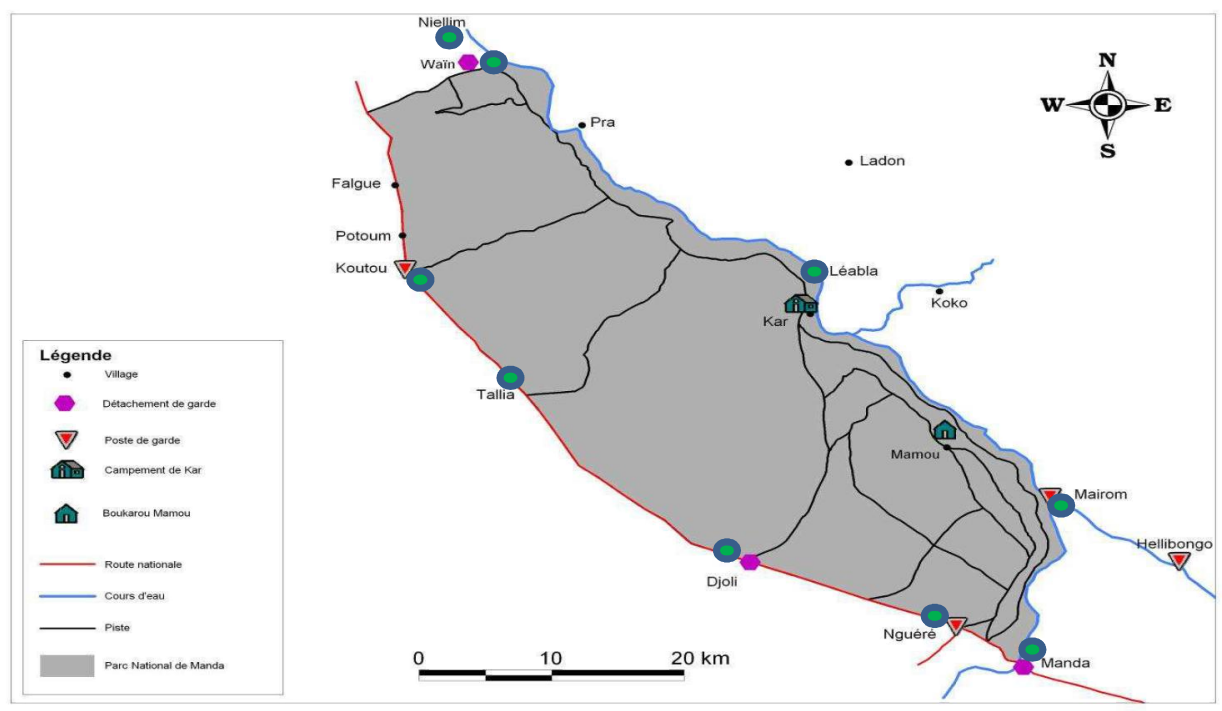

Localisation des villages retenus pour les études

Figure 2 : Carte de localisation des villages d'étude autour du PNM. 


\section{RESULTATS}

Les informations recueillies auprès des habitants de ces villages d'études, les fonctionnaires des services déconcentrés, les observations de terrain et les images satellitaires ont permis d'identifier les facteurs déstructurant des écosystèmes parmi lesquels l'effet de la délocalisation des villages à la création de l'aire protégée. Cet effet est suivi de celui d'exploitation des ressources et enfin l'effet d'induction.

\section{Effets de la délocalisation des villages ou effet de report}

Un an après la création du PNM, plusieurs villages ont été délocalisés en 1966. Parmi ceux-ci figurent les villages de Gori, Hôle, Teblai, Kö̈n, Kaako, Koutou, Falké, Waïn, Sanglet, Doboro, Maïbo, Doguigui, Beboro, Djoli1, Djoli2, Nangnda, Guéré, Kemata, Yanga, Nangnda II, Goumkou, Yan, Dobadi, Baadon....

Ces villages ont été délocalisés d'une manière peu orthodoxe, si bien que beaucoup d'entre eux se sont retrouvés du côté ouest du parc sans aucune planification préalable. Cette délocalisation non planifiée a dû modifier par ricochet le schéma d'accès aux ressources naturelles.

En effet, dans la zone d'étude, le choix de l'espace agricole d'une famille, par exemple doit se faire de manière à ne pas empêcher à une autre famille la possibilité d'augmenter leur surface culturale les prochaines années, si elle la souhaite. L'alignement des champs des familles doit être plus ou moins parallèles ou comme des rayons qui partent d'une même source lumineuse ; une famille n'a pas droit de placer son champ juste au-dessus d'un champ d'une autre famille. Le schéma généralement admis est celui de la Figure 3.

Sur cette Figure 3, les orientations des flèches bleues correspondent à l'alignement des champs des familles du village généralement admis mais la flèche de couleur rouge, placée au bout d'une flèche bleue n'est pas acceptée ; car l'emplacement d'un champ de cette manière, est proscrit. Un tel emplacement empêche la famille dont le champ est situé à la base de la flèche rouge d'augmenter sa surface agricole mais le rapprochement des villages dû à la délocalisation va changer ce modèle ancien d'accès aux ressources.

\section{Modification du modèle d'accès aux ressources}

La Figure 4 de petite échelle met en exergue le phénomène de rapprochement des villages explicités.

En outre, la gestion circonstancielle des terres oblige les paysans à ne plus respecter le schéma habituel (Figure 5) d'accès aux ressources. La Figure 5 illustre parfaitement la nouvelle approche d'accès engendrée par la création de l'aire protégée.

Cette nouvelle approche d'accès a pour conséquence la surexploitation des terres provoquant des dégradations $\mathrm{du}$ couvert végétal. Les bosquets et les galeries forestières ont fortement régressé sous la pression exercée par l'abus de leur utilisation dans la zone périphérique. Cette situation est d'autant plus remarquable dans les villages de Sanglet, Doboro, Maïbo, Doguigui et Beboro qui n'ont pas de nos jours des bosquets denses. La Figure 5 qui montre l'emplacement de ces villages est illustrative. Les images satellitaires ont mis en exergue le changement de structure des écosystèmes au fil du temps. Certains écosystèmes cèdent de la place à d'autres dans les sens vertical et horizontal sous les effets de l'exploitation de leurs composantes.

\section{Effets d'exploitation}

$\mathrm{La}$ délocalisation des villages occasionnée par la création du parc a provoqué un «effet de report». Fort de ce qui précède, l'image satellitaire de l'année 1985 (met en exergue une savane arborée aussi importante à l'intérieur du parc qu'à l'extérieur, même si la densité végétale semble un peu plus élevée dans le parc. A la périphérie, l'espace est dominé par les cultures dans le canton Balimba suivi du 
canton Djoli puis le canton Kokaga. La superficie des cultures dans le canton Niellim est de moindre envergure. Dans le même canton, on observe de relique de forêt claire à Falgué et dans le canton Djoli. Cette forêt claire observée dans le canton Djoli correspond à la forêt classée de Djoli-Kera, un autre espace protégé contigu au parc national de Manda.

Il apparaît de manière assez évidente que le nombre des strates verticales dans les espaces périphériques sur lesquels sont concentrés les champs est réduit aux strates cryptogamique et herbacée et dans certaine mesure à une strate arbustive.

La situation s'aggrave plus tard en 1995 en ce sens que la savane arborée ne se remarque dans le canton Djoli non loin des villages Sandana, à Gayam, Koro, Maïbo, Maïnadja et un tout petit peu à Falgué dans le canton Niellim au croisement des deux cantons (Djoli et Niellim). Même si la strate arborée semble céder de la place dans les cantons Djoli et Balimba, elle en est gagnée dans les cantons Niellim et Kokaga comme nous pouvons observer sur l'image satellitaire.

Cette situation va s'accentuer dix ans plus tard en 2005. La savane arborée a régressé à la hauteur des villages Mabanon et Sandoro. La forêt claire et la savane arborée observées sur l'image de 1995 ont totalement disparu (voir les flèches sur la Figure 8), faisant place aux cultures et à la savane arbustive. La forêt claire n'est observée en dehors du parc que dans le canton Niellim sur l'autre rive du fleuve Chari. Les forêts galeries sont également remarquées dans le même canton et dans le canton Kokaga.

\section{Effets d'induction}

Entendons par effets d'induction les effets qui ne sont pas directement provoqués par la création du parc mais engendrés au cours du temps par la gestion des ressources hors parc et qui peuvent avoir des portées lointaines.

\section{Substitution floristique}

Fort de ce qui précède, on assiste à une substitution végétale. Sur des terres fortement dégradées se développent des espèces indicatrices des sols infertiles localement connues comme Striga hermontica ( $t$ Dro» en Sara Madjingaye), Calotropis procera («ndöbӘ»), Guiera senegalensis ( kambӘnda»). Ces espèces énumérées ont remplacé celles à croissance lente notamment les Humenocardia acida ("kokar»), Gardenia ternifolia ( massi»), Landolphia owariensis (Apocynaceae, liane, ("douï» en Sara Madjingaye avec un ton de prononciation bas)), Strychnos spinosa (Loganiaceae, arbuste, ( douï » en Sara Madjingaye avec un ton de prononciation haut)). Les autres espèces végétales ont diminué considérablement en fréquence à cause de leur forte sollicitation dans les usages quotidiens. Il s'agit notamment de Cymbopogon giganteus (Poaceae, ( karwaïn»), Andropogon gayanus (poaceae, herbe, ("wandog»)), Acacia ataxacantha (Mimosaceae, ( ( ngär»)), Jardinea congoensis (Poaceae, herbe, («tӘba»)), Bambusa vulgaris (" kagӘnal »).

Tentatives de restauration de certaines espèces végétales

Pour faire face à cette rareté, il y a des paysans qui ont opté pour la culture de certaines espèces en l'occurrence Cymbopogon giganteus, une herbe d'une taille de 2 à $3 \mathrm{~m}$ dans le tissage de seko et nattes. Cette restauration a commencé avec l'initiative d'un paysan de village de Boundourou dans le canton Djoli. Ce paysan en 2004 a décidé de cultiver cette espèce sauvage. Ensuite les habitants des villages environnants (Kana, Talia, Bodo, Sandoro, Bita, Namo, Rekmadji, etc.) ont emboîté le pas. D'autres villages ont préféré la culture de Bambusa vulgaris (bambou) pour son utilisation dans la construction des habitats.

Mosaïque d'aires protégées dues à la création des zones de mises en défens

Ces communautés ne se sont pas arrêtées là. Avec l'appui technique et financier 
d'un projet de développement dénommé «Projet de Conservation et Utilisation Durable de la Biodiversité dans le Moyen Chari », elles ont créé des zones de mises en défens (Figure 12) pour essayer de gérer rationnellement le reste de leurs ressources disponibles. Ces zones de mises en défens ont été créées par canton. Certains cantons ont choisi de faire de réserves de forêts communautaires comme c'est le cas des cantons Kokaga et Balimba. Le canton Djoli, à défaut de bosquet dense, a plutôt créé une forêt sacrée pour les rites initiatiques des jeunes adolescents. Le canton Niellim a préféré une zone de mise en défens halieutique pour contrôler les pêches et laisser le temps aux alevins d'atteindre la maturité afin d'assurer la pérennité des ressources ichtyologiques. Cette initiative de mise en défens de certaines ressources a suscité l'engouement des autres cantons proches mais non limitrophes du parc national de Manda à créer leurs zones de mise en défens. Ce sont les cantons Bedaya, Bessada et Dobo. A l'instar des autres cantons, après élaboration d'un plan de développement local et plusieurs rencontres d'échanges avec les cantons riverains au parc, deux réserves forestières et une zone de mise en défens halieutique ont été créées respectivement dans les cantons Bessada, Dobo et Bedaya.

La création de ces zones de mise en défens autour du parc national de Manda crée donc une mosaïque d'aires protégées (Figure 12) donnant une différente structure horizontale d'écosystèmes, ce que Dajoz R. appelle hétérogénéité spatiale de la biocénose. La Figure 12 présente les détails.

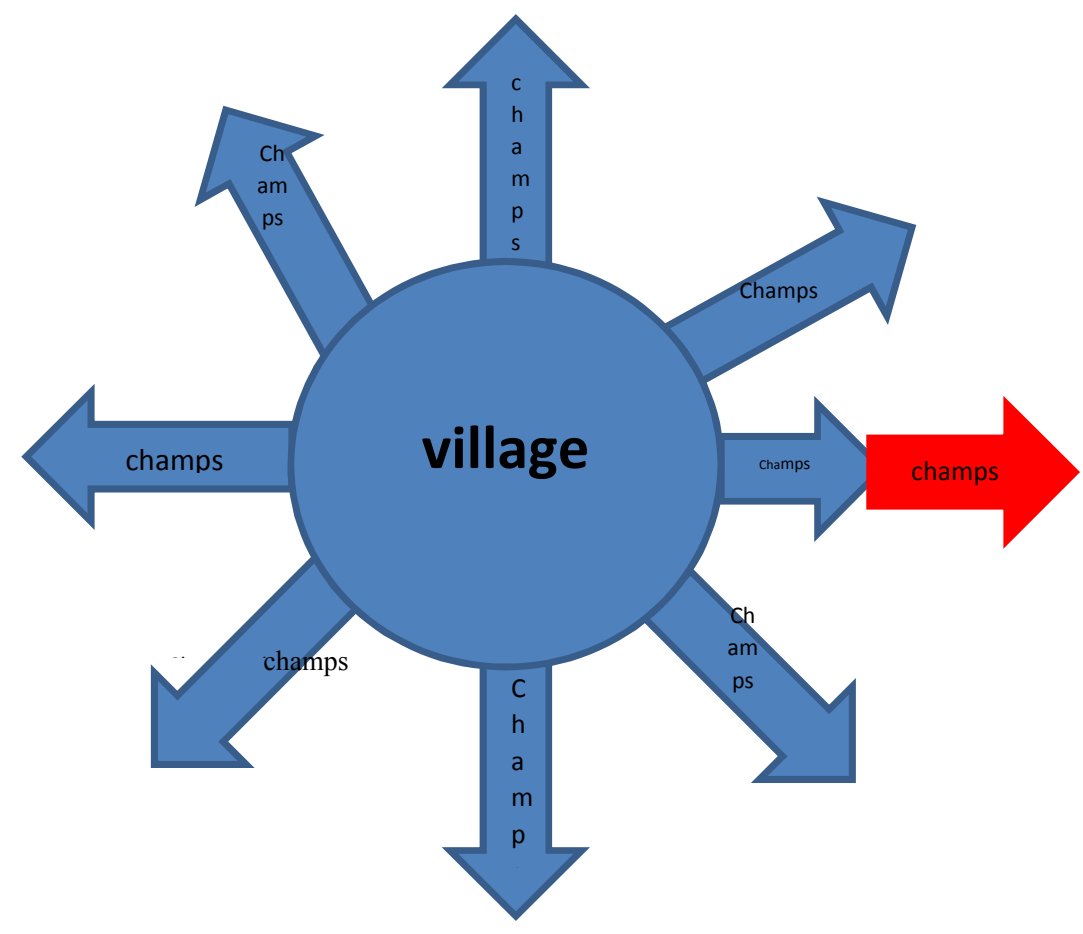

Figure 3 : Schéma d'alignement préféré des champs des villageois au Sud du Tchad. 


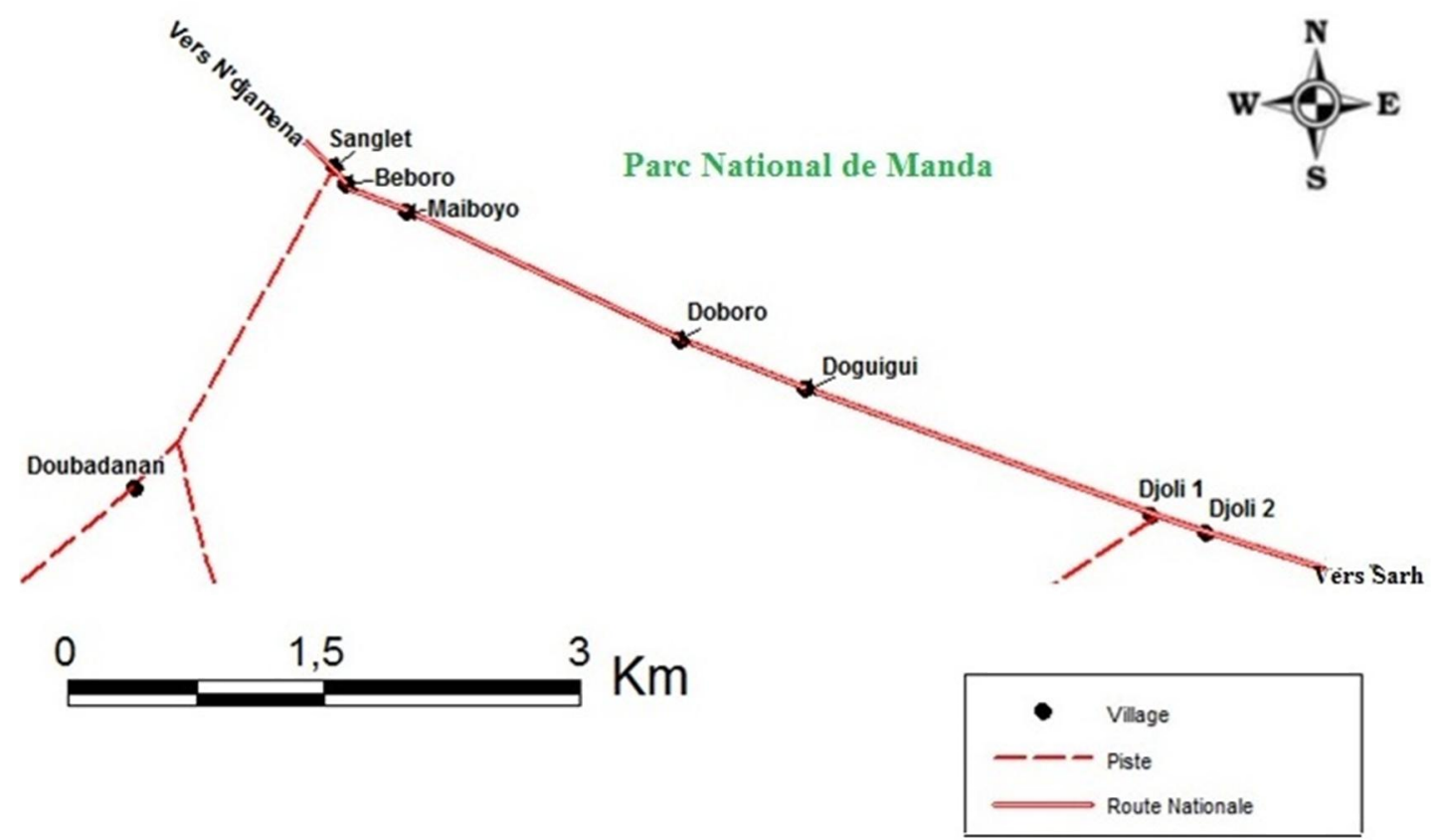

Figure 4 : Rapprochement des villages suite à la délocalisation.

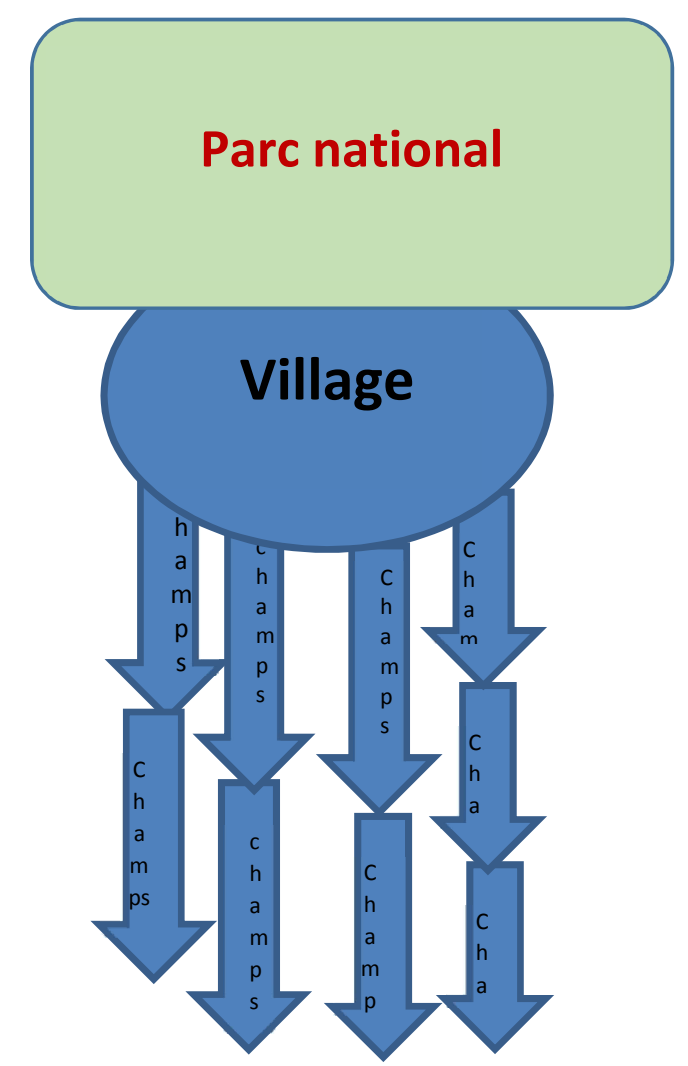

Figure 5 : Schéma d'alignement des champs des villageois modifié par la création du parc. 
$18^{\circ} 0^{\circ} 0^{\prime \prime E}$

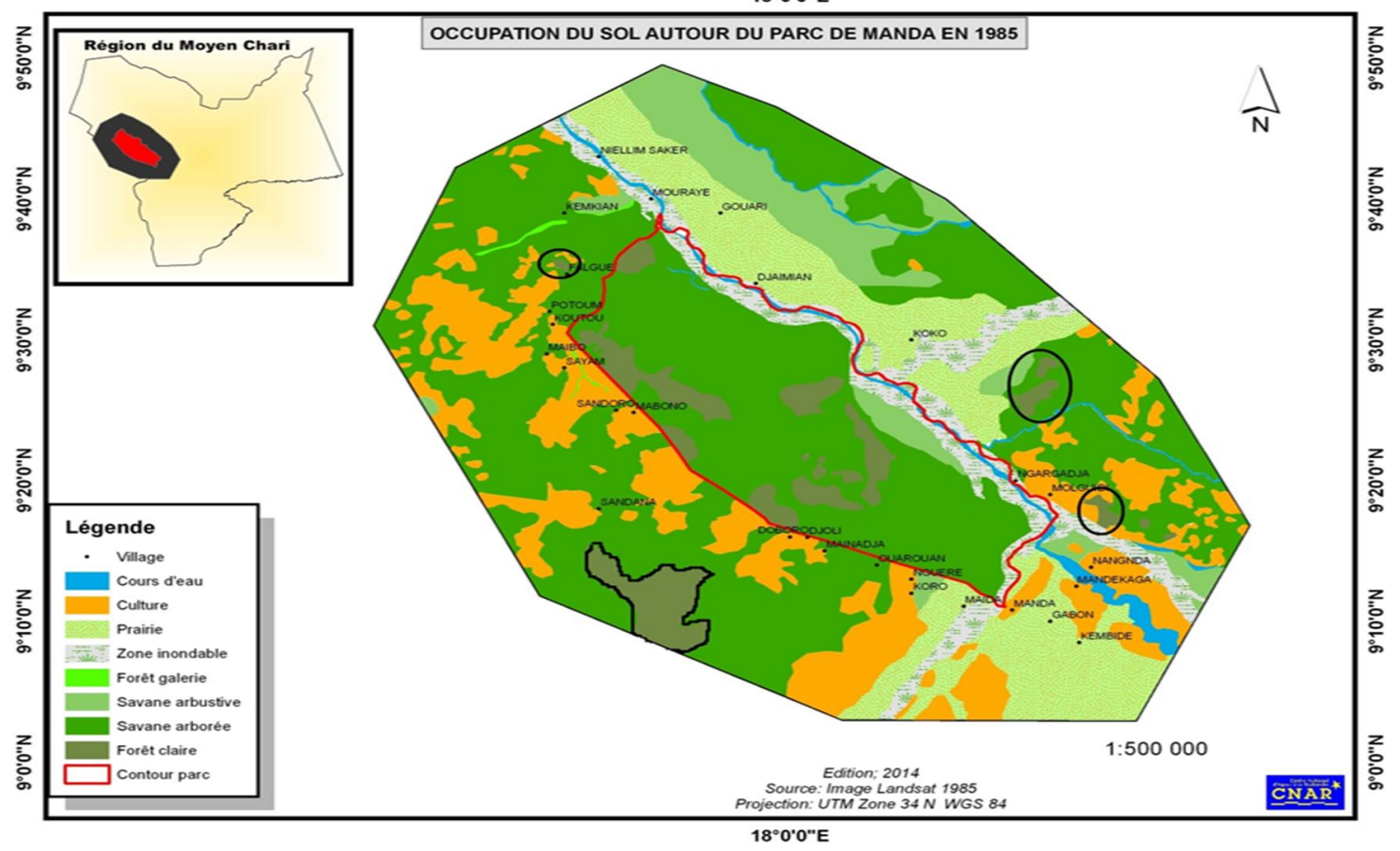

Figure 6 : Occupation du sol autour du parc en 1985.

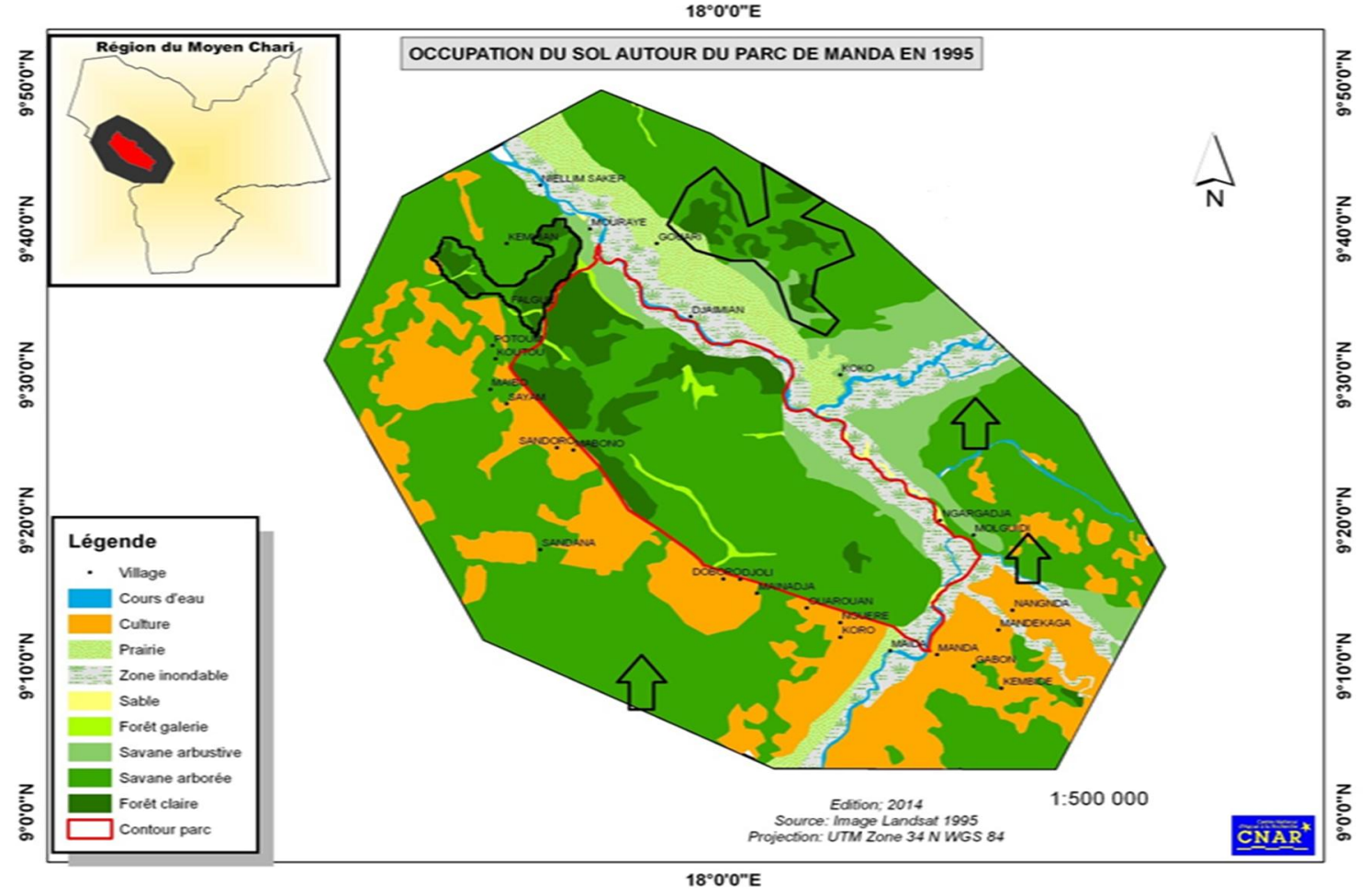

Figure 7 : Occupation du sol autour du parc en 1995. 
$18^{\circ} 0^{\prime} 0^{\prime \prime}$

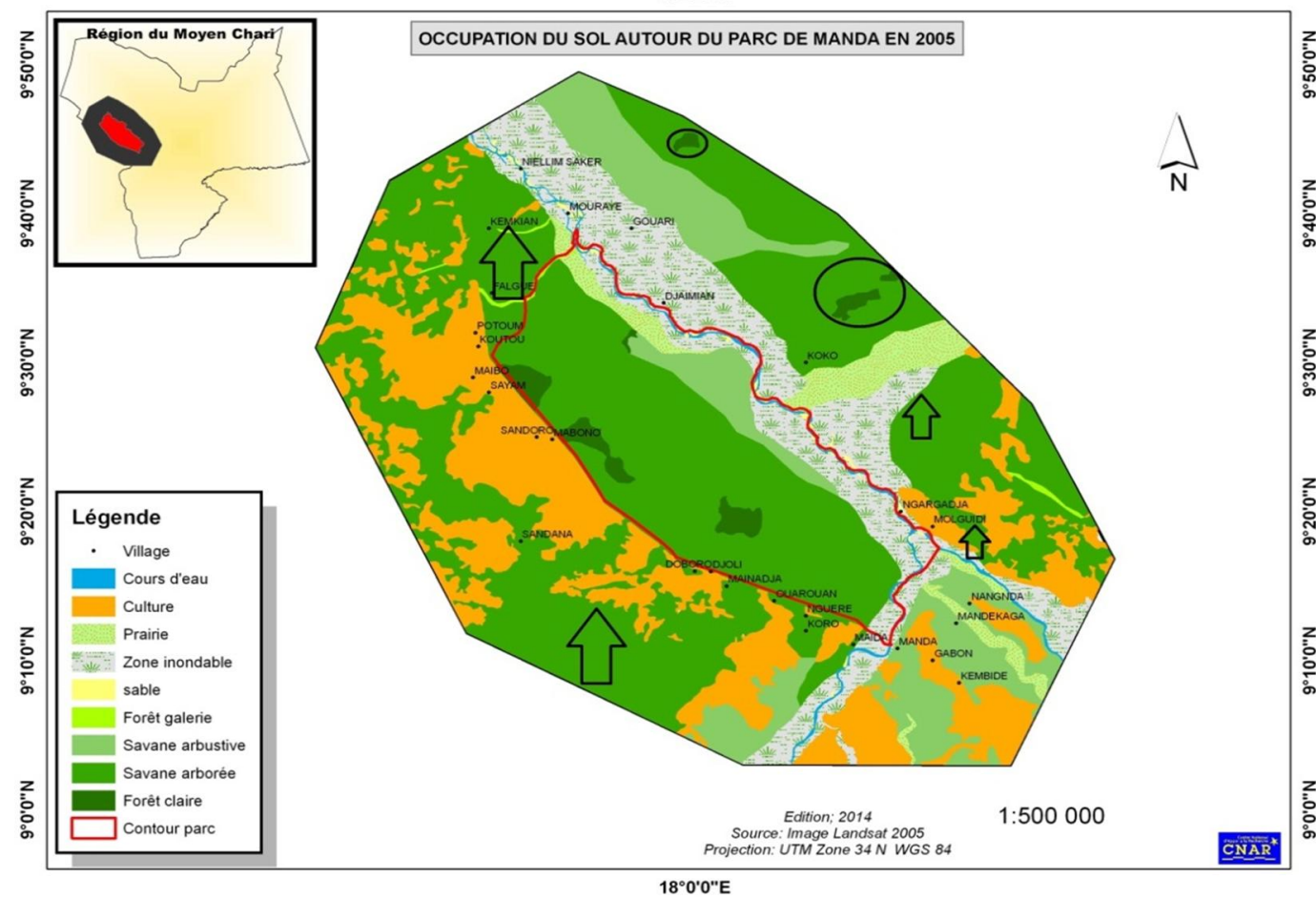

Figure 8 : Occupation du sol autour du parc en 2005.

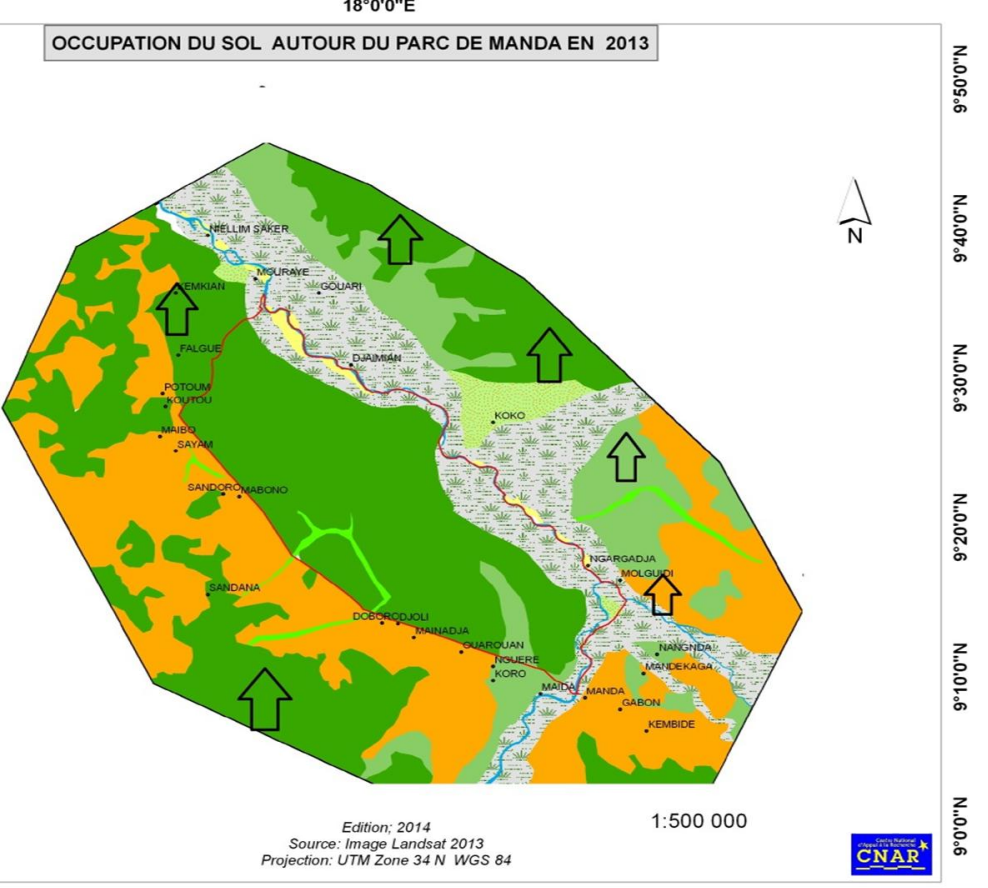

Figure 9 : Occupation du sol autour du parc en 2013. 


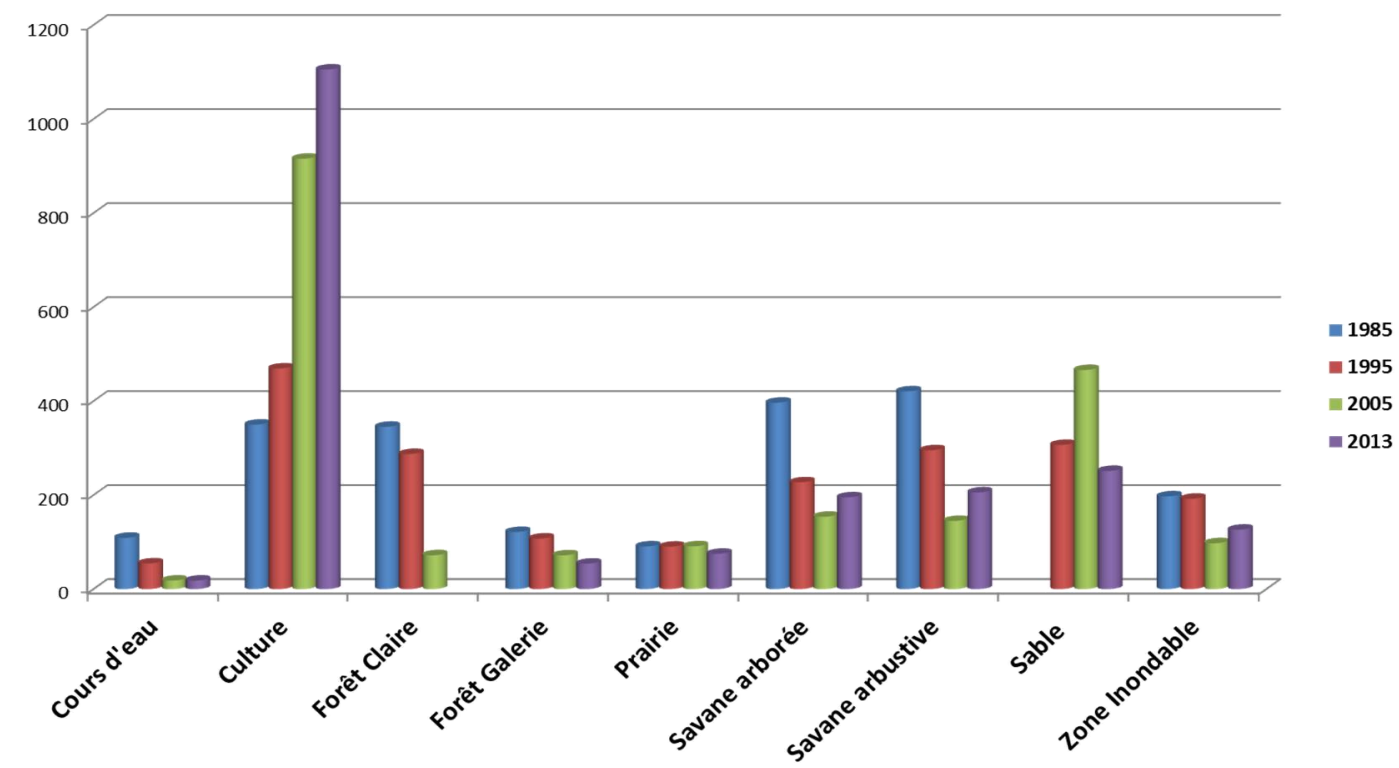

Figure 10 : Cumul des unités d'occupation de sol autour du PNM de 1985 - 2013.

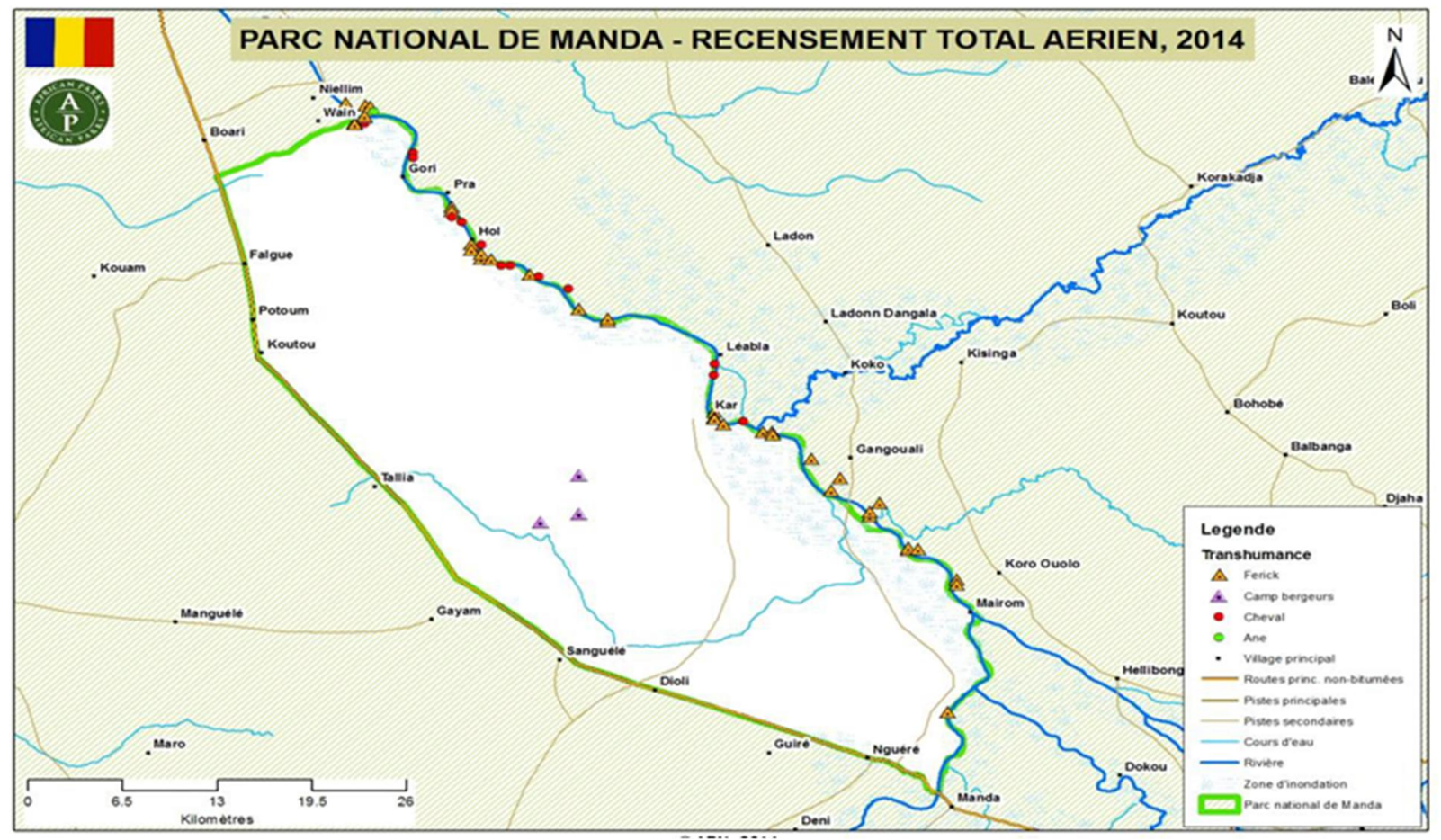

Figure 11 : Carte de localisation des campements des éleveurs et pêcheurs. 


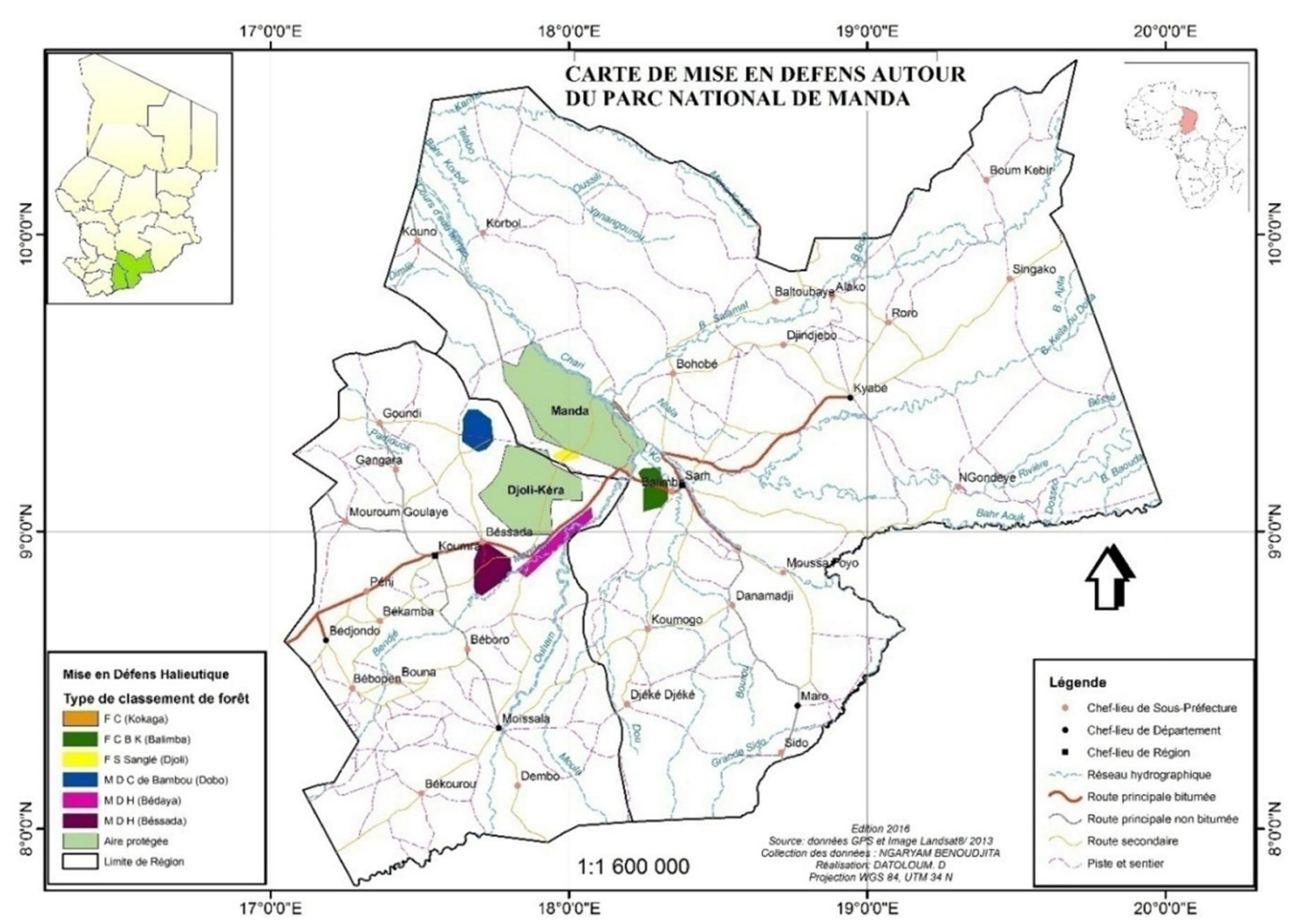

Figure 12 : Carte de localisation de la mosaïque des aires protégées.

\section{DISCUSSION}

Les impacts générés par la création du Parc National de Manda sont occasionnés majoritairement par la délocalisation des villages pour une proportion de 41,6\%. Cette délocalisation s'est traduite dans les faits par une surexploitation des ressources. A cela s'ajoute l'effet de report à $37 \%$ qui se justifie par le retrait de certaines ressources de l'utilisation habituelle. Les effets d'induction qui sont de 21,4\% se résument aux différents impacts qui produisent d'autres impacts.

Concrètement, la création de cette aire protégée a opéré un changement profond dans le schéma d'alignement anciennement préféré (Figure 3) des champs des villageois pour deux raisons principales qui sont :
- Le rapprochement des villages délocalisés sans mesures d'accompagnement et sans étude préalable de relocalisation ;

- La restriction de la surface cultivable car une grande partie de l'espace communautaire est désormais sous protection.

Une délocalisation négociée et préalablement planifiée avec toutes les parties prenantes (Dan Guimbo et al., 2012 ; Sandjong Sani et al., 2013) permettraient d'identifier les sites de relocalisation des villages concernés de manière à laisser des grands espaces exploitables entre les villages contrairement à ce qu'on remarque sur la Figure 4. Ce manque de planification et de concertation est donc déterminant dans le rapprochement des villages Sanglet, Beboro, Maiboyo, Doboro, Doguigui, Djoli1 et Djoli2 
déplacés suite à la création du Parc National de Manda. Selon les récits des habitants questionnés, ces villages qui étaient jadis situés à des bonnes distances l'un de l'autre selon le schéma local d'occupation de l'espace. Le rapprochement des villages délocalisés à la création du Parc National de Manda, a contraint l'exploitation des ressources naturelles exclusivement de côtés Ouest et Sud du parc rendant la forêt qui était autrefois dense selon les images satellitaires étaient devenues claires. Car à défaut d'avoir accès à des nouvelles terres agricoles fertiles, les paysans se rabattent aussitôt sur des terres en jachère raccourcissant d'année en année la période de jachère. Les causes de cette dégradation sont donc l'absence de régénération, l'exploitation abusive du bois et des produits non ligneux, la sécheresse et l'insuffisance des pluies (Dan Guimbo et al., 2012). L'irrégularité des pluies dans la zone d'étude est un facteur qui n'est pas lié à la création du parc mais elle a un impact d'importance majeure sur la régénération des plantes. Par conséquent, elle reste déterminante dans la célérité des mutations observées dans la structure des écosystèmes. Les changements climatiques se traduisent par une mortalité anormalement accrue des arbres (Sandjong Sani et al., 2013). Le retour de la forêt claire dans ces zones s'explique par une bonne pluviométrie en 1995 selon les données satellitaires (Figure 7). Autrement dit, le climat a joué un rôle considérable sur la végétation de la zone voisine du PNM. D'autres facteurs ne sont pas à perdre de vue. Le feu de brousse, par exemple, est récurent dans la zone soudano-sahélienne (Inouss et al., 2014) à cause de l'aridité des milieux et les pratiques de chasse.

Ces différents facteurs ont changé les structures verticales et horizontales des écosystèmes. De la structure verticale à 4 strates végétales à savoir la strate cryptogamique, la strate herbacée, la strate arbustive et la strate arborescente ou arbustive
(Faurie et al., 2012), l'on est passé à deux strates ou trois selon les endroits et l'année abondamment pluvieuse ou non (Figures 9 et 10). L'exploitation des ressources qui devrait se faire de manière parsemée ou uniforme en fonction de localisation géographique des villages avant la création du parc selon les personnes enquêtées, est concentrée dans certains endroits et cela favorise les plantes envahissantes ou de régénération rapide (Tiebre et al., 2014 ). Cette destruction des écosystèmes périphériques ne s'exprime pas de manière uniforme.

Il convient de souligner que la dégradation des ressources ne se manifeste pas, non plus, immédiatement suite à la création de l'aire protégée mais c'est un processus qui se met en place au fil du temps et dépendant de la densité démographique et de l'espace hors parc disponible. La proximité avec certaines agglomérations urbaines l'influence fortement pour des raisons d'empreinte écologique de celles-ci.

L'image satellitaire de 2013, révèle que le temps est un facteur déterminant dans le changement de structure des écosystèmes sous l'effet des activités anthropiques. Les quelques reliques de forêts claires ont totalement disparu des zones périphériques de l'aire protégée. Les flèches de la carte 9 montrent l'emplacement des forêts claires disparues.

Les données numériques fournies par satellite donnent une représentation graphique ci-dessous (Figure 10) qui permet de se rendre effectivement compte du changement; certains types d'écosystèmes ont cédé de la place aux autres (Avakoudjo, 2014). Sur cette Figure 10, l'on remarque une augmentation de la superficie des cultures $(54,42 \%$ en 2005$)$ au détriment des forêts claires $(3,54 \%$ en 2005$)$ qui ont régressé jusqu'à disparaitre en 2013.

Comme précédemment élucidé, la délocalisation des villages suivie de leur rapprochement ne sont pas les seuls facteurs qui expliquent la dégradation des écosystèmes 
adjacents du Parc National de Manda. En effet, la création de ce parc a attiré beaucoup d'éleveurs transhumants et les pêcheurs d'autres régions du pays par la disponibilité des ressources offertes par le parc (Ngaryam, 2016). La densité de la population qui est de 8,7 habitants $/ \mathrm{km}^{2}$ sur le plan national en 2009 , est de 21,82 habitants $/ \mathrm{km}^{2}$ dans la zone d'étude pour la même année. Autrement dit, la démographie a galopé avec la création du parc.

En outre, le nombre de têtes de bétails a considérablement augmenté. Monnery dans une étude qui date de 1997, a fait état d'une augmentation « de plus de $422 \%$ de bovins et plus de $14,6 \%$ de petits ruminants dans la zone ». L'objectif visé par les éleveurs par leur présence massive, est de laisser leurs bétails paitre dans le parc (Lesse et al., 2015). A défaut d'y pénétrer à cause de la surveillance renforcée, ces animaux domestiques se concentrent sur les zones périphériques augmentant par ricochet la pression sur les ressources des zones périphériques (Djirra, 2011; Avakoudjo, 2014). Les bétails broutent les herbes dans les zones adjacentes au parc; le piétinement du couvert végétal provoque une dégradation. En plus, ces pasteurs transhumants se sédentarisent de plus en plus en associant la culture à l'élevage (Manceron, 2011). Ce qui pose deux problèmes majeurs :

- Premièrement, l'accès à l'espace agricole ;

- Deuxièmement, un terrain d'habitation devant servir à construire des cases d'hébergement appelé en arabe local « férick 》 (Figure 11).

En sus de la pression déjà exercée sur les ressources par leurs bétails précédemment élucidée, leur sédentarisation suivie d'une deuxième activité constituent une pression supplémentaire (Lesse et al., 2015, Ngaryam, 2016). Ainsi l'espace agricole restreint par la création de l'aire protégée fait l'objet de convoitise des deux grands acteurs: autochtones et nomades attirés par les ressources du parc et qui finissent par échouer dans les zones périphériques et devenant par la suite cultivateurs ou agro-éleveurs (Avakoudjo, 2014).

Au total, 38 féricks et 32 campements de pêcheurs (Figure 11) ont été dénombrés en 2014 autour du parc par l'équipe de «projet Biodiversité ». La Figure 11 donne une idée sur les installations de ces féricks et campements aux alentours du Parc National de Manda. L'attrait des éleveurs transhumants par les aires protégées est exprimé par Manceron (2011) dans la périphérie du parc du W en Afrique de l'Ouest. Houehanou et al. (2008) ont exprimé la même préoccupation dans la gestion pastorale et la structure des terroirs agricoles de la périphérie de Djona (Nord-Est Bénin).

Parmi ces espèces végétales qui sont rares ou ayant disparues des zones périphériques, on peut distinguer celles fréquemment sollicitées dans les usages et celles à croissance lente qui n'ont pu résister à la concurrence des plantes envahissantes (Tiebre, 2014). Les paysans riverains au parc étaient dans l'obligation de cultiver certaines espèces végétales d'utilité courante disparues dans leurs zones. Ces exemples mettent en visibilité le changement de perception des paysans par rapport aux ressources naturelles. Ils rejoignent au gré des circonstances Lévêque (2010) qui disait: "on ne s'était guère préoccupé du devenir de la diversité biologique, aussi longtemps que les ressources vivantes paraissaient inépuisables, et que l'espace était suffisant pour permettre à l'homme d'occuper de nouvelles terres, sans compromettre pour autant l'avenir des autres espèces ». Ils ont cru à l'inépuisabilité des ressources mais ils sont rattrapés par l'idée exprimée par Arne Næss que «la perception qu'on a du monde, forme le fondement de notre irrespect à l'encontre de la nature ». La rareté de certaines ressources a conduit certes, à une prise de conscience de la communauté locale avec une tentative de restauration de 
certaines espèces disparues mais elle n'occulte pour autant pas le système de gestion de la biodiversité remis en cause. La création des aires protégées de nos jours dans le but de conserver durablement la biodiversité, devrait se faire de manière à ne pas ruiner rapidement les ressources hors parc. Une vision synoptique est un gage de durabilité.

\section{Conclusion}

Bien que les écosystèmes soient naturellement dans un état de perturbation continuelle sous l'action des facteurs écologiques, le changement de structure explicitée dans cette étude résulte en grande partie, de la création d'une aire protégée. Le Parc National de Manda a changé fortement la structure des écosystèmes périphériques à cause de l'effet de report dû à la mise sous cloche d'un grand espace comme réserve. Cette restriction est suivie d'une délocalisation forcée non organisée et mal planifiée sur un petit espace. En sus la démographie, l'agriculture et le surpâturage ont engendré une concentration d'exploitation des ressources sur un petit espace d'accès libre provoquant une dégradation des écosystèmes. Certaines strates végétales comme la strate arborée a disparu par endroit ainsi que la strate arbustive. La dégradation n'a pas seulement modifié la structure écosystémique mais elle a un effet considérable sur le changement de peuplement végétal. Les espèces végétales à croissance lente et exigeant en élément nutritifs ont cédé de la place aux espèces à croissance rapide et envahissantes capable de se développer sur des substrats infertiles. Le corollaire est que ce phénomène a réduit la richesse de la biodiversité des zones périphériques conduisant à la création d'autres types d'aires protégées à gestion communautaire connues sous les termes «zone de mise en défens ». La présence de celles-ci présente une mosaïque autour du parc national de Manda donnant en vue aérienne des écosystèmes protégés dans un état acceptable à proximité des écosystèmes fortement dégradés car sous pression anthropique. En outre, la stratification de la faune est aussi touchée car les strates végétales dégradées constituent les habitats des animaux. La composante faunistique de la biocénose ne souffre pas seulement du manque d'habitat mais le braconnage est un facteur déterminant à la réduction de leur nombre.

Il convient de relever que le changement de structure et du peuplement des écosystèmes des zones périphériques d'une aire protégée s'exprime en fonction de la dimension d'espace d'accès libre, de la densité démographique, de la manière dont les communautés riveraines exploitent les ressources de leur localité et surtout des conditions de la création de l'aire protégée.

\section{CONFLIT D'INTERETS}

Les auteurs déclarent qu'ils n'ont aucun conflit d'intérêts dans le cadre du présent manuscrit.

\section{CONTRIBUTIONS DES AUTEURS}

NB est intervenu dans toutes les phases de l'étude. Il s'agit de la conception de l'étude, la collecte des données, le dépouillement, le traitement et l'analyse des données et la rédaction du présent manuscrit. ADI est intervenu dans le traitement et l'analyse des données et dans la correction du manuscrit.

\section{REMERCIEMENTS}

Ce travail a été réalisé avec l'accord institutionnel de l'Ecole Normale Supérieure de Bongor au Tchad. Nous exprimons notre grande reconnaissance à cette institution universitaire. Notre gratitude s'oriente aussi vers toutes les personnes qui, nous ont aidés dans la collecte des données de terrain, notamment les agents de services publics en charge de l'eau, de l'environnement et des forêts, de l'agriculture et de l'élevage. Nous 
remercions aussi les habitants des villages riverains au parc qui nous ont accompagnés efficacement.

\section{REFERENCES}

Arne N. 2008. Ecologie, Communauté et Style de Vie. MF Dehors : Paris ; 373.

Arnould P, Simon L. 2007. Géographie de l'Environnement. Atouts Géographie: Belin, Paris ; 303p.

Aissatou TN, Ousmane N, Moustapha BS, Aly D, Didier G. 2015. Caractérisation de la végétation ligneuse sahélienne du Sénégal: cas du Ferlo. International Journal of Biological and Chemical Sciences, 9(6):2582-2594.

Avakoudjo J, Mama A, Toko I, Valentin K, Sinsin B. 2014. Dynamique de l'occupation du sol dans le Parc National $\mathrm{du} \mathrm{W}$ et sa périphérie au nord-ouest du Bénin. International Journal of Biological and Chemical Sciences, 8(6): 2608-2625.

Barbault R, Bonneau L, Constantin F, Corbier C, Gerbe P, Gouin R, Kleitz G, Leblanc E, Robinet O, St Martin G, Vernet P, Weber J, 2004. Des espaces protégés pour concilier la biodiversité et le développement, Paris, $60 \mathrm{p}$.

Dajoz R. 2006, Précis d'Ecologie (8 ${ }^{\mathrm{ème}}$ édn). Dunod : Paris ; 631.

Desponds D. 2009. Les impacts d'un parc naturel régional (PNR) sur les évolutions socio-démographiques de son espace rural : le cas du Vexin français ", Norois [En ligne], 202 | 2007/1, mis en ligne le 01 mars 2009, consulté le 11 avril 2012. http://norois.revues.org/1620

Djirra HB. 2011. Influence des pratiques agropastorales sur la végétation dans et autour du parc national de manda, Mémoire de Master, Université de Sarh, CREFELD, Tchad, 66 p.

Dovonou-Vinagbè P, Chouinard O. 2009. Gestion communautaire des ressources naturelles au Bénin (Afrique de l'Ouest)
: le cas de la vallée du Sitatunga ", Études caribéennes [En ligne], 12 | Avril 2009, mis en ligne le 19 juillet 2009, consulté le 08 avril 2014. URL : http://etudescaribeennes.revues.org/3630 ; DOI : 10.4000/etudescaribeennes.3630

Dufumier M.. « Biodiversité et agricultures paysannes des Tiers-Mondes », Annales de géographie, 2006/5 $\mathrm{n}^{\circ} 651$, p. 550568. DOI : 10.3917/ag.651.0550

Faurie C., Ferra C., Médori P., Dévaux J., Hemptinne J-L., 2012. Ecologie approche scientifique et pratique, 6è éd., Lavoisier, Paris, 488 p.

Gauthier-Clerc M., Mesléard F., Blondel J., 2014. Sciences de l'environnement, de boeck, Paris, Bruxelles, 346 p.

Giraut F., Guyot S. et Houssay-holzschuch M.. « Les aires protégées dans les recompositions territoriales africaines», article en ligne en 2004 sur : halshs.archives-ouvertes.fr/docs/00/ 18/56/81/PDF/IG.pdf.

Gunnell Y. 2009. Ecologie et société : repères pour comprendre les questions de l'environnement, Armand Colin, Paris, $415 \mathrm{p}$.

Houehanou TD, Houinato M, Adandedjan C, Gbangboche AB, Hounzangbe-adote MS, Sinsin BA. 2008. Gestion pastorale et structure des terroirs agricoles dans la périphérie de la Djona (Nord-Est Bénin). International Journal of Biological and Chemical Sciences, 2(4): 497-507.

Inoussa MM, DIOUF A, Bakasso Y, Morou B, Zaman-allah M, Mahamane A, Saadou M. Situation de référence de la phytodiversité et la productivité herbacée d'un dispositif de suivi du feu de brousse au Niger. International Journal of Biological and Chemical Sciences, 8(3): 1165-1178.

Lesse P, Houinato MRB, Djenontin J, Dossa H, Yabi B, Toko I, Tente B, Sinsin B. 2015. Transhumance en République du Bénin : états des lieux et contraintes. 
International Journal of Biological and Chemical Sciences, 9(5): 2668-2681.

Lévêque C. 2010. Biodiversité : réflexion et controverses sur une nature en danger. Les causes de l'érosion de la biodiversité;1-15. http://www. futurasciences.com/ magazines/ environnement/ infos/dossiers/ $\mathrm{d} /$ developpement- durable-biodiversite.

Manceron S. 2011. Intervenir en périphérie pour la conservation des aires protégées : réexamen d'un postulat - la situation du parc du W et des éleveurs mobiles. Thèse de doctorat, Université Paris 10, Nanterre la Défense, $586 \mathrm{p}$.

Ngaryam B. 2016. Problématique de gestion durable de la biodiversité au Tchad: impacts des aires protégées sur les zones périphériques - cas des parcs nationaux de Manda et de Sena oura ». Thèse de doctorat Université Paris 8, Saint Denis, $365 \mathrm{p}$.

Ramade F. 2005. Eléments d'Ecologie Ecologie Appliquée (6 ${ }^{\mathrm{e}}$ édn). Dunod: Paris ; 864.

Sandjong sani RC, Ntoupka M, Ibrahima A, Vroumsia T. 2013. Etude écologique du Parc National de Mozogo-Gokoro (Cameroun): prospections préliminaires de la flore ligneuse et du sol pour sa conservation et son aménagement. International Journal of Biological and Chemical Sciences, 7(6): 2434-2449.

Tiebre M-S, Kouame Dj, Vroh ATB, N'da D. K, Adou Y-CY. 2014. Stratégies et potentiel d'invasion des massifs forestiers par Hopea odorata Roxb. (Dipterocarpaceae) : cas du Parc National du Banco en Côte d'Ivoire. International Journal of Biological and Chemical Sciences, 8(2): 666-679. 